\title{
CHRISTOPHE IPPOLITO, Vers des identités culturelles postfrancophones
}

\section{Alexandre Calvanese}

\section{(2) OpenEdition}

\section{Journals}

\section{Édition électronique}

URL : https://journals.openedition.org/studifrancesi/45499

DOI : 10.4000/studifrancesi.45499

ISSN : 2427-5856

\section{Éditeur}

Rosenberg \& Sellier

\section{Édition imprimée}

Date de publication : 1 août 2021

Pagination : 406-408

ISSN : 0039-2944

\section{Référence électronique}

Alexandre Calvanese, "CHRISTOPHE IPPOLITO, Vers des identités culturelles postfrancophones », Studi Francesi [En ligne], 194 (LXV | II) | 2021, mis en ligne le 01 septembre 2021, consulté le 15 octobre 2022. URL : http://journals.openedition.org/studifrancesi/45499; DOI : https://doi.org/10.4000/ studifrancesi.45499

Ce document a été généré automatiquement le 15 octobre 2022

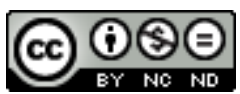

Creative Commons - Attribution - Pas d'Utilisation Commerciale - Pas de Modification 4.0 International - CC BY-NC-ND 4.0

https://creativecommons.org/licenses/by-nc-nd/4.0/ 


\title{
CHRISTOPHE IPPOLITO, Vers des identités culturelles postfrancophones
}

\author{
Alexandre Calvanese
}

\section{RÉFÉRENCE}

CHRISTOPHE IPPOLITO, Vers des identités culturelles postfrancophones, Caen, Passages, 2019, $280 \mathrm{pp}$.

1 L'auteur réunit dans cet ouvrage une série d'études qui avaient déjà été présentées (sous une forme qui a pu être, entre-temps, plus ou moins remaniée en vue de la présente publication) en revue ou en volume entre 2004 et 2017. L'hétérogénéité des sujets abordés s'explique par la pluralité des intérêts de recherche qui ont caractérisé le parcours de l'auteur au cours des années, ce qui n'empêche pas d'y retrouver un fil rouge, à savoir l'exigence, évoquée dans le titre, d'interroger «les identités culturelles plurielles des littératures dites francophones» (p. 9). Mais que veut signifier, au juste, l'adoption du préfixe post, qui n'est pas sans évoquer d'autres catégories littéraires comme celles de postmoderne ou de postcolonial? IPPOLITo ne le précise que dans la conclusion de son ouvrage (pp. 231-243), comme s'il avait voulu d'abord démontrer la nécessité d'aller au-delà du concept de francophonie, sans que cela implique la volonté de l'effacer radicalement. En effet, si dans l'introduction (pp. 9-30) il rappelle les limites, les contradictions et les ambiguïtés qui ont marqué l'emploi de ce terme (avec ou sans majuscule) aussi bien dans le domaine politique (responsable d'une véritable pollution du mot, p. 17) que dans celui plus spécifiquement littéraire, et si les six chapitres (regroupés en trois parties) qui composent le livre sont autant d'exemples qui invitent à remettre en question l'opacité de cette notion, voilà enfin explicité l'horizon de sa démarche: «Le terme de postfrancophonie lui-même n'est pas appelé à remplacer de façon permanente celui de francophonie, qu'on voudrait voir revenir, loin de la politique, loin des discriminations, à son sens purement littéral, mais à lui opposer un concurrent, à temporairement signaler la nécessité d'une critique de la notion de 
francophonie (dans ses dérives officielles et utilitaires), plus de dix ans après le manifeste pour une littérature-monde» (p. 241).

2 La première partie, intitulée «Pouvoirs du faux», s'interroge sur la capacité de la littérature de créer des identités fictives, illusoires, ou de travailler pour promouvoir des identités culturelles et politiques qui n'existent pas encore.

3 Le premier chapitre (pp. 33-68), en examinant l'inscription de la dimension spectaculaire d'Impression d'Afrique de Raymond Roussel dans L'Afrique fantôme de Michel Leiris, s'attache à montrer que l'Afrique a souvent été, pour les Européens, l'objet d'une mise en scène qui en dit plus du sujet qu'elle représente que de l'objet représenté. Cette analyse se compose de trois moments.

Dans un premier temps Ippolito propose un répertoire des axes thématiques autour desquels se regroupent, selon Leiris, «des pratiques d'écriture, des opérations, des stratégies qui produisent la représentation spectaculaire» (p. 35) chez l'auteur des Impressions d'Afrique: il s'agit des modalités subjectives d'appropriation du réel (principalement le fétichisme et le sadomasochisme); de la transformation du rapport au réel poursuivie à travers la création du folklore, de mythes, de légendes, d'une dimension magique; de la dimension ludique et dramatique de la représentation, élément incontournable dans un roman qui, selon la critique Annie Le Brun, «est si peu un roman qu'il raconte un spectacle qui va se transformer en pièce de théâtre» (p. 36).

5 Dans un deuxième moment, Ippolito examine de quelle manière ces mêmes axes influencent la composition de L'Afrique fantôme de Leiris, avec le résultat qu'une "œuvre née dans un cadre scientifique [...] se teint [...] d'une couleur littéraire» (p. 50) et qu'elle «se fait déborder par le réel, surtout dans la disparate première partie» (p. 51). Sans vouloir à tout prix déclarer «l'influence décisive» du récit de Roussel sur celui de Leiris, pour Ippolito «il reste que les deux œuvres sont placées sous le signe du spectaculaire (sujet de Roussel et objet de Leiris), et qu'elles s'éclairent l'une l'autre en ce sens», mais surtout que «Leiris à Gondar, et Roussel à Paris, trouvent quelque chose qui n'est pas l'Afrique et qui est plus que l'Afrique, un espace et un temps immobiles où peuvent sans cesse se perpétuer les spectacles» (p. 58).

6 Dans un troisième moment, enfin, l'auteur se propose de montrer de quelle manière L'Afrique fantôme se construit par un rapport d'imitation/opposition envers deux genres - le journal intime et le journal de voyage - et d'y explorer la fonction jouée par le rituel et par sa mise en scène, capable de sublimer et de fondre, dans cette œuvre, l'ethnographie et la littérature.

7 Le deuxième chapitre (pp. 69-108) se concentre sur deux passages importants de la construction d'une identité libano-francophone. Le premier correspond à la courte mais décisive existence de la "Revue Phénicienne": courte car sa parution ne dura qu'un semestre (les quatre numéros sortirent entre juillet et décembre 1919); décisive en relation au rôle joué par l'élite économique et culturelle maronite qui anima la revue dans le processus qui aboutira, en septembre 1920, à la création du «Grand Liban» par la France (qui conservera son mandat jusqu'en 1946). Après avoir rappelé le contexte historique de l'après-guerre, et les différents acteurs impliqués dans la région, Ippolito explore les contenus de la revue: des éditoriaux de Charles Corm (directeur de la revue et en même temps d'une maison de commerce à Beyrouth) qui profita de cette tribune pour réclamer l'appui de la France, aux articles qui insistèrent sur les 
différences historiques et politiques entre Syrie et Liban et qui firent écho à un discours guerrier et nationaliste nourri, entre autres, par le mythe phénicien fondateur.

Le deuxième moment analysé dans ce chapitre prend en compte la reconstruction du champ francophone libanais dix ans après la fin de la guerre civile (1975-1989), notamment à travers une étude du marché de l'édition en langue française et des mécanismes de légitimation des écrivains libanais francophones. S'appuyant sur des rapports produits par le Syndicat national de l'édition (1986) et par le Sénat français (2001), Ippolito met en évidence les retombées de la politique de coopération et des projets d'aide à l'édition (comme le soutien aux publications du "Centre d'études et de recherches sur le Moyen Orient contemporain"), tout en reconnaissant que «l'effort français, si important qu'il soit, resterait artificiel sans les efforts locaux, tels ceux de Dar An-Nahar, maison d'édition libanaise» (p. 88). Cependant, remarque Ippolito, la dépendance par rapport à la France constitue un indéniable point de faiblesse, car «la littérarité n'est souvent légitimée [...] que par ricochet (prix littéraire à Paris, etc...)» (p. 94). C'est alors dans le but de rééquilibrer au profit du camp libanais les relations culturelles avec la France qu'il faut interpréter la création de la collection «Patrimoine» par Dar An-Nahar: l'objectif principal de cette «Pléiade» libanaise inaugurée en 1986 (avec toujours le support du Service du Livre français au Liban) et interrompue en 2002 (par manque de budget: une réflexion sur la viabilité des initiatives nées grâce à l'appui de la coopération s'impose...) a été en effet de faire connaître les auteurs libanais de langue française, pas seulement ceux qui (comme Nadia Tuéni ou Georges Schéhadé) avaient déjà obtenu une consécration internationale, mais aussi ceux qui ont été oubliés après avoir joué un rôle dans la culture libanaise, et dont les ouvrages, «souvent introuvables ailleurs [...], peuvent être considérés comme des archives de la "libanofrancophonie" classique» (p. 108).

9 La deuxième partie du livre, intitulée «Identités culturelles postfrancophones en mouvement», s'ouvre avec un chapitre (pp. 111-138) entièrement consacré à la poétesse libanaise Nadia Tueni. Dans un premier temps, Ippolito revient sur son expérience en tant qu'éditeur scientifique d'une co-édition bilingue (française et anglaise) et binationale (Liban et États-Unis) de deux recueils de poèmes de Tueni: Liban: Vingt poèmes pour un amour, paru en 1979 chez Dar An-Nahar et Archives sentimentales d'une guerre au Liban, paru en 1982 chez Jean-Jacques Pauvert, ont été réunis (quoique seulement de manière partielle pour ce qui concerne le deuxième) dans Lebanon: Poems of Love and War / Liban: Poèmes d'amour et de guerre, paru en 2006 conjointement pour les presses de l'Université de Syracuse et pour Dar An-Nahar. Ces pages mettent en relief l'effort de négociation demandé par une initiative éditoriale de ce genre, aussi bien sur le plan de la traduction (par exemple dans le choix de gommer ou de reproduire un marqueur de différence culturelle) que sur celui de l'édition (par exemple dans le choix des traducteurs et des auteurs des essais critiques qui accompagnent les poèmes, mais aussi dans la présentation de certains événements historiques et politiques).

Dans la deuxième partie du chapitre, Ippolito propose une analyse du recueil Archives sentimentales d'une guerre au Liban, écrit par Tueni pendant la guerre civile libanaise et lit cet ouvrage comme l'aboutissement d'un parcours poétique conçu comme engagement contre la propagande idéologique marquée par la voix des hommes, un parcours dans lequel «l'équivalence métaphorique [...] entre femme et pays» constitue la principale invariante du langage de Tueni: «elle crée dans ses poèmes des "archives" 
sentimentales de son pays en guerre, établissant une "mémoire" qui prend la forme d'un cordon ombilical étendu entre le sujet poétique et la terre» (p. 137).

11 Le quatrième chapitre (pp. 139-166) interroge les concepts d'identité migrante et de diaspora, d'exil et d'errance, de nostalgie et de mémoire dans le roman La Québécoite (1983) de Régine Robin. C'est peut-être le chapitre dans lequel on ressent le plus le fait que ce livre recueille des essais composés de manière indépendante les uns par rapport aux autres, car les deux parties qui le composent sont par moments redondantes, et elles auraient probablement profité d'une refonte intégrale en un discours plus organique et linéaire, compte tenu aussi de la complexité du texte analysé. Parmi les nombreux nœuds thématiques mis en relief par Ippolito dans le roman de Robin, il nous semble intéressant de mettre en relief - en relation au sujet des identités culturelles postfrancophones - cette partie de son analyse qui porte sur la démystification de toute conception essentialiste d'idées telles que "tradition", "origine", "identité": "Ce que conteste en fait Robin est l'idée moderniste d'une nostalgie des origines (d'un "je me souviens" univoque et désespéré), enracinée dans les mythes fondateurs et l'histoire commune qu'avec les années les Québécois, "immigrants comme les autres", ont eu le temps de constituer» (p. 160), tandis que sa narratrice revendique sa condition de "québecoite», c'est-à-dire de migrante, d'exilée, de nomade. «Robin problématise l'histoire du Québec français en montrant les limites $\mathrm{du}$ projet souverainiste de réécriture de l'histoire, [...] l'aveugle adhésion au conformisme nationaliste dont le besoin illusoire est une conséquence malheureuse mais directe de l'histoire des Québécois en tant que minorité canadienne qui vise à se reconstruire en peuple» (ibidem).

La troisième partie du livre, dont le titre «Que peut la postfrancophonie littéraire?» est à lui-même une référence intertextuelle, s'ouvre avec un chapitre (pp. 169-203) conçu comme une contribution à «la lente constitution d'un corpus de textes qui remplace le vieux corpus scolaire franco-français» (p. 169); ce but est poursuivi à travers une analyse des liens, aussi bien intratextuels qu'intertextuels, qui participent à la richesse (la "valeur ajoutée qu'est la littérarité») de deux œuvres de Léon Gontran Damas et Marie NDiaye. Dans le cas du premier, Ippolito s'intéresse au recueil poétique Névralgies (1972 pour l'édition définitive), et en particulier à l'intégration dans ce dernier du précédent recueil Graffiti (1952): persuadé que «[1]es combinaisons de variantes dans les poèmes contribuent à démultiplier les changements de sens» (p. 184), l'auteur passe d'abord en revue les correspondances sémantiques dans les titres des deux recueils et les variations dans l'ordre des poèmes (d'un recueil à l'autre), pour analyser ensuite les modifications internes (à chaque poème), comme la «réorganisation généralisée de groupes de vers» (p. 182) ou les «changements sémantiques déterminants» (p. 184). L'attention à la structure interne de l'œuvre n'empêche pas de mettre en lumière le dialogue que Damas a entretenu avec d'autres auteurs, en particulier avec les poètes surréalistes. En ce qui concerne Marie NDiaye, Ippolito esquisse une lecture intertextuelle de Trois femmes puissantes (2009) de l'écrivaine sénégalaise et des Trois contes (1877) de Gustave Flaubert, considérant «ces deux séries [comme] un collage de six récits dont plusieurs éléments s'imbriquent et migrent vers les autres» (p. 203). Le but de ce chapitre est plus de contester l'arbitraire du découpage français/francophone que de mettre en relation les deux textes, par exemple en montrant la manière dont NDiaye aurait retravaillé certains éléments des récits de Flaubert (cet aspect étant limité à quelques observations sur le rapport entre Rudy et Khady et leurs "modèles" 
flaubertiens Julien et Félicité, ou sur le décor symbolique évoqué dans les deux ouvrages).

Le sixième et dernier chapitre (pp. 205-229) se donne pour objectif de «mettre en avant une postfrancophonie mondialisée et "heureuse" de l'être» (p. 205), c'est-à-dire capable de profiter de certains aspects de la mondialisation - avant tout la facilitation des relations interculturelles permise par la toile et ses moyens de communication - pour déjouer le joug (néo)colonial. Dans un premier temps il s'agit de réfléchir sur la possibilité d'adapter des expériences réussies dans le domaine de l'édition francophone - Ippolito revient ici sur le cas du Liban - au contexte africain. Ce qui ressort de l'analyse est cependant que le rôle de la coopération française (ou internationale) reste quasiment indispensable pour que le marché de l'édition africaine francophone puisse sortir du mécanisme par lequel le succès d'un livre en Afrique dépend essentiellement de son succès à Paris: et donc support dans l'événementiel (organisation de salons du livre), dans la diffusion et publication locales, mais aussi aide aux centres de recherche. Quant à la possibilité de répliquer une initiative comme la collection «Patrimoine» de l'éditeur libanais Dar An-Nahar, pour Ippolito elle implique la nécessité d'établir une liste des auteurs et des œuvres classiques pour chaque pays africain francophone. Pour l'auteur, «adopter une stratégie globale à l'échelle de l'ensemble des pays francophones du continent pour "décoloniser" l'édition francophone africaine semble irréaliste, et ne tient pas compte de la diversité que le colonialisme et le néo-colonialisme ont tenté de gommer» (p. 210); il s'agit plutôt de promouvoir une démarche de légitimation et de réappropriation $\mathrm{du}$ patrimoine sur le plan national, visant une «action culturelle identitaire» (p. 212).

Dans la dernière partie, le chapitre présente le blog de Muzdalifa House, un centre culturel ouvert en 2009 à Moroni, aux Comores. Soeuf Elbadawi est le principal animateur du centre et l'auteur principal du blog; il se bat pour la décolonisation de Mayotte (département et région française depuis 2011) et sa réintégration politique dans l'archipel des Comores, une fracture qui, entre autres choses, a provoqué la mort de plus de 8000 migrants dans le bras de mer qui sépare l'île d'Anjouan de Mayotte. Le blog devient ainsi un instrument de résistance culturelle, un «vecteur de diffusion et de propagation de l'action militante», «l'expression d'une conscience collective» (p. 222), «archipel de textes qui affirme l'unité de la nation et de la culture comoriennes et ce faisant pose un problème universel et lancinant: Qu'est-ce que les régressions coloniales aujourd'hui? Quelles formes prennent-elles? Comment dessiner des résistances?» (p. 228). 